\title{
Optimum-Interval Punched-Card Tables
}

This paper describes a new principle in table-making that leads to greater efficiency in punched-card applications. Tables of the ordinary kind, proceeding by equal intervals of the argument, are of ten much more extensive than is necessary. In the new tables the number of entries is reduced to the minimum by allowing the interval of the argument to vary and by modifying the tabulated quantities so that interpolation is still performed in the usual way.

The use of tables in computations that are made by means of punched cards usually entails the following operations: ${ }^{1}$ the detail cards are sorted into the table cards according to the left-hand part of the argument, the function and first difference are gang-punched from the table cards onto the detail cards, and the interpolation of the function to the value corresponding to the complete argument is accomplished by one operation of the form $F \pm n d$, with the multiplying punch. This assumes that the table is sufficiently extensive so that second differences are negligible.

In some cases a less extensive table, used with an interpolation formula that includes second differences, may be more efficient. The necessity of deriving coefficients of the second difference may be avoided if the differences in the table are replaced by the coefficients of the powers of $n$ in the interpolation formula. Thus Bessel's formula becomes

$$
\begin{aligned}
F & =F_{0}+n \Delta_{1}{ }^{\prime}+\frac{n(n-1)}{4}\left({\Delta_{0}}^{\prime \prime}+{\Delta_{1}}^{\prime \prime}\right) \\
& =F_{0}+n\left(D_{1}+n D_{2}\right),
\end{aligned}
$$

where $D_{1}=\Delta_{1}^{\prime}-\frac{1}{4}\left(\Delta_{0}^{\prime \prime}+\Delta_{1}^{\prime \prime}\right), D_{2}=\frac{1}{4}\left(\Delta_{0}^{\prime \prime}+\Delta_{1}^{\prime \prime}\right)$ are the quantities to be tabulated instead of the customary first and second differences. This principle can easily be extended to higher orders of differences.

The method just described is most efficient in application when the second differences are large and when the third differences may be neglected. When the contribution of the second differences becomes small it becomes relatively wasteful to take account of it in this way. Sometimes the difficulty may be overcome by some approximate method of sorting and gangpunching, but this is time-consuming and subject to error. The ordinary alternative is to extend the argument one more decimal place and to increase the number of cards in the table tenfold, a procedure which is even more wasteful and inefficient.

Our purpose here is to describe a much more powerful expedient, the optimum-interval method. The interval of the argument is allowed to vary throughout the table, the criterion being that it shall be just small enough at every point so that linear interpolation will be legitimate, with the result that the number of cards in the table is greatly reduced. This advantage is obtained without complicating the use of the table in any way, and in spite of the unusual tabular intervals.

In using any ordinary table the interpolating factor, $n$, is obtained by subtracting the tabular argument from the given argument, and by dividing 
this difference by the tabular interval of the argument. When the tabular interval is an integral power of 10 , then $n$ is simply those figures of the complete argument beyond the position corresponding to the last digit of the tabulated argument. When the interval of the argument is not an integral power of 10 , let us suppose all the differences of the table to be divided by the interval of the argument. Form the product of this modified difference by those figures of the complete argument beyond and including the position corresponding to the last digit of the tabulated argument. If this product is added to the function, the result differs from the correct interpolate by an amount equal to the product of the modified difference times the last figure of the tabulated argument. By modifying each function in the table to the extent of deducting this product, the resulting table will admit of direct interpolation in the usual manner in spite of the odd interval; it is only necessary to retain one more figure in the multiplier.

The value of the interval, $\omega$, is determined by the equation $\Delta^{\prime \prime}=\omega^{2} d^{2} f / d x^{2}$, where $\Delta^{\prime \prime}=4$, if the neglected second difference effect is not to exceed onehalf unit. The sequence of intervals must be chosen so that every argument the last figure of which is zero is retained in the table. Otherwise there will be cases in which the full amount by which the function has been modified will not be restored. Of all the possible combinations of intervals, the following four (in addition to the unit interval) are all that it is necessary to employ in order to obtain the advantages of the method:2, 2, 2, 2, 2; $2,3,2,3 ; 3,3,4 ; 5,5$.

A table of this kind differs from the ordinary table in respect of the accuracy attainable with a given number of figures. If the quantities in an ordinary table are accurate to $0.5 \mathrm{unit}$ and if the second differences are negligible it is well known that a rounded-off interpolate has a maximum error of 1.0. The differences in the modified table are multiplied by factors larger than unity and the error of the interpolate depends on the interval of tabulation. The maximum rounding-off error is $\frac{1}{2}(1+p / q)$ units, where $p$ is the largest interval of the argument and $q$ is the unit of the argument. In many applications an error of this size is not important but if it can not be tolerated the remedy is to carry the values of the modified functions and differences to one more significant figure. In punched-card applications the additional figure usually does not complicate the use of the table or reduce the efficiency.

To illustrate the advantages of optimum-interval tables we have prepared a six-place table of reciprocals, portions of which are shown below. The table is actually carried to seven places in order to insure six-place accuracy in the interpolates, and the tabular intervals have been chosen so as to keep the maximum error due to neglect of the second difference less than 5 units of the seventh place. Since the function is $x^{-1}, \Delta^{\prime \prime}=2 \omega^{2} x^{-3}=4$ or $x^{3}=\omega^{2} / 2$. Taking $\omega=2,3,4,5,10,20,30,40$, in succession, the corresponding values of the argument at which these intervals may be employed are, with sufficient approximation, 1.260, 1.660, 2.000, 2.320, 3.700, 6.000, $7.700,9.300$, respectively. Let us consider a given argument lying between 1264 and 1266. In the unmodified table it is necessary first to subtract 1264 from the given argument and to divide by 2 in order to obtain the interpolating factor $n$. In the modified table the differences are already divided by the interval. If the end figures of the argument beyond 126 are used as 
an interpolating factor the result will be in error by $4 D$, and this amount has been subtracted algebraically from the true reciprocal of 1264 in order to obtain the function of the modified table. Thus the reciprocal of 12655 is $7936393-5.5 \times 6249=790202$. It is advisable in practice to add 5 to every entry of the table in order to provide for automatic rounding-off of the interpolates to six figures and this has been done here.

The total number of cards in the table of reciprocals is 1368 , as compared with 9000 in the usual table, thus affording a saving of $2 \frac{1}{2}$ hours of sorting and gang-punching each time the table is used. The loss due to an extra figure in the multiplier is one hour for each 10,000 detail cards. In the range from 1000 to 3700 and 370 to 600 , the multiplier contains all the figures of the argument beyond the third figure, and from 600 to 999 it contains all beyond the second. In the range from 370 to 600 , the modified table does not differ from an ordinary table.

Another example of the improved efficiency possible with the use of optimum-interval tables is a table of seven-place sines and cosines. The usual table, with a tabular interval of 0.01 , contains 9000 cards. The modified table contains 2700 cards, with intervals $0: 03,0.03,00^{\circ} 04$. The maximum contribution of the neglected second difference is 0.6 unit of the seventh decimal. Each time this table is used, more than two hours of sorting and gang-punching time is saved.

The application of this principle will be found of value in all cases of punched-card tables that differ considerably from linearity over the whole range of the argument.

\begin{tabular}{|c|c|c|c|c|}
\hline Arg. & Unmod & able & Modif & \\
\hline$x$ & $1 / x$ & $-\Delta^{\prime}$ & $f$ & $-D$ \\
\hline $\begin{array}{l}1250 \\
1251 \\
1252 \\
1253 \\
1254 \\
1255 \\
1256 \\
1257 \\
1258 \\
1259 \\
1260 \\
1262 \\
1264 \\
1266 \\
1268 \\
1270\end{array}$ & $\begin{array}{l}8000005 \\
7993610 \\
7987225 \\
7980851 \\
7974487 \\
7968132 \\
7961788 \\
7955454 \\
7949131 \\
7942817 \\
7936513 \\
7923935 \\
7911397 \\
7898899 \\
7886440 \\
7874021\end{array}$ & $\begin{array}{l}6395 \\
6385 \\
6374 \\
5364 \\
6355 \\
6344 \\
6334 \\
6323 \\
6314 \\
6304 \\
12578 \\
12538 \\
12498 \\
12459 \\
12419 \\
12381\end{array}$ & $\begin{array}{l}8000005 \\
7999995 \\
7999973 \\
7999943 \\
7999907 \\
7999852 \\
7999792 \\
7999715 \\
7999643 \\
7999553 \\
7936513 \\
7936473 \\
7936393 \\
7936279 \\
7936120 \\
7874021\end{array}$ & $\begin{array}{l}6395 \\
6385 \\
6374 \\
6364 \\
6355 \\
6344 \\
6334 \\
6323 \\
6314 \\
6304 \\
6289 \\
6269 \\
6249 \\
6230 \\
6210 \\
6191\end{array}$ \\
\hline $\begin{array}{l}1990 \\
1992 \\
1995 \\
1997 \\
2000 \\
2003 \\
2006 \\
2010\end{array}$ & $\begin{array}{l}5025131 \\
5020085 \\
5012536 \\
5007516 \\
5000005 \\
4992516 \\
4985050 \\
4975129\end{array}$ & $\begin{array}{l}5046 \\
7549 \\
5020 \\
7511 \\
7489 \\
7466 \\
9921 \\
7414\end{array}$ & $\begin{array}{l}5025131 \\
5025117 \\
5025086 \\
5025044 \\
5000005 \\
4999983 \\
4999930 \\
4975129\end{array}$ & $\begin{array}{l}2523 \\
2516 \\
2510 \\
2504 \\
2496 \\
2489 \\
2480 \\
2471\end{array}$ \\
\hline & 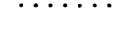 & & & . \\
\hline $\begin{array}{l}3690 \\
3695 \\
370\end{array}$ & $\begin{array}{l}2710032 \\
2706365 \\
2702708\end{array}$ & $\begin{array}{l}3667 \\
3657 \\
7285\end{array}$ & $\begin{array}{l}2710032 \\
2710020 \\
2702708\end{array}$ & $\begin{array}{r}733 \\
731 \\
7285\end{array}$ \\
\hline
\end{tabular}




\begin{tabular}{ccccc} 
Arg. & \multicolumn{2}{c}{ Unmodified Table } & \multicolumn{2}{c}{ Modified Table } \\
$\boldsymbol{x}$ & $1 / \boldsymbol{x}$ & $-\Delta^{\prime}$ & $f$ & $-D$ \\
371 & 2695423 & 7246 & 2695423 & 7246 \\
$\ldots$ & $\ldots \ldots \ldots$ & $\ldots \ldots$ & $\ldots \ldots \ldots$ & $\ldots \ldots$ \\
599 & 1675047 & 2801 & 1675047 & 2801 \\
598 & 1672246 & 2792 & 1672246 & 2792 \\
599 & 1669454 & 2782 & 1669454 & 2782 \\
600 & 1666672 & 5537 & 1666672 & 2769 \\
602 & 1661135 & 5501 & 1666637 & 2751 \\
604 & 1655634 & 5464 & 1666562 & 2732 \\
606 & 1650170 & 5428 & 1666454 & 2714 \\
608 & 1644742 & 5393 & 1666318 & 2697 \\
610 & 1639349 & 5357 & 1639349 & 2679
\end{tabular}

U. S. Naval Observatory, Washington, D. C.

1 Compare W. J. Eckert, Punched Card Methods in Scientific Computation, New York, The Thomas J. Watson Astronomical Computing Bureau, Columbia University, 1940.

\section{RECENT MATHEMATICAL TABLES}

138[A].-Mathematical Tables Project, New York, A. N. Lowan, technical director, Table of Reciprocals of the Integers from 100,000 through 200,009. New York, Columbia University Press, 1943, viii, 201 p. $19.6 \times 26.5 \mathrm{~cm}$. Reproduced by a photo offset process. $\$ 4.00$.

This work is designed to provide a 7-place table of reciprocals between 100,000 and 200,000 , wiich will "expand by tenfold the scope of the existing tables in this interval."

The table is presented in what James Henderson has called the "modern arrangement" where the first five figures of the argument proceed in natural order down the page and the final figures from 0 to 9 are given along the top. Only the significant figures in the reciprocals are recorded. Since the entries are close together the differences vary from 100 at the beginning of the table to 25 at the end. Tables of proportional parts are given at the bottom of each page.

According to the preface we learn that: "Preparation of manuscript tables was begun in December, 1934, by Dr. C. C. Kiess of the National Bureau of Standards. The work was completed under his direction in May, 1939, with the co-operation, at various times, of Messrs. B. F. Scribner, H. R. Mullin, W. G. Esmond, and J. Waldron."

It may be well to add a short account of the development of tables of reciprocals, which have been in the making for more than a century. The first adequate table appears to have been that of PETER BARLOw, published in the first edition of his Tables in 1814 [MTAC, p. 17], where the reciprocals are given for the first 10,000 integers. A. De Morgan, in the edition of 1840 , says: "I cannot ascertain that any tables of square roots, cube roots or reciprocals comparable in extent to those of Mr. Barlow were ever printed before his." In the fourth edition, issued in 1941 by L. J. Comrie, the reciprocals were extended from 10,000 to 12,500 . Comrie found " 60 errors of a unit in the last decimal, but none greater" in the 1840 edition.

The next significant contribution was R. PICARTE's, La division réduite à une Addition, Paris, 1861, which provided values of the reciprocals from 1000 to 10,000 to 10 significant figures, together with the first nine multiples of them.

W. H. OAKEs published his 7-place Table of the Reciprocals of Numbers from 1 to 100,000, in London in 1865. The volume under review is a continuation of this work, which was similarly printed in the "modern arrangement," with differences at the side of the page. The more recent table of M. B. CorsworTH, erroneously entitled "Reciprocals for All Numbers from 1 to $10,000,000, "{ }^{1}$ is also a 7 -figure table over the same range as that of Oakes. 